\title{
NOUVEaUX cas humains de fasciolase hépatiQue AU PORTUGAL
}

\author{
Par Fernand FONSECA, J. FRAGA DE AZEVEDO \\ et M. MARQUÉS DA GAMA
}

Nous avons publié en 1948, dans cette revue (Ann. Paras. hum. et comp., $23: 18,1948$ ), le premier cas humain de fasciolase hépatique signalé au Portugal. Depuis cette date, six nouveaux cas de la même parasitose ont été enregistrés par nous, auxquels il faut ajouter encore cinq cas rapportés par Célestino Maia dans l'île de Madère (1951).

En face de ces exemples, nous pouvons conclure qu'au Portugal, comme dans beaucoup d'autres pays, nous sommes en présence d'un accroissement de cette maladie.

Parmi les cas que nous avons décrits, provenant de plusieurs endroits du pays, trois se rapportaient à des hommes et trois à des femmes, tous âgés de 27 à 45 ans. Récemment, nous avons pris connaissance de deux cas, dont l'un concernait une femme de 37 ans et l'autre un individu de 22 ans. Nous nous référons également à eux dans ce travail.

En général, la maladie durait quelques mois ou années et était toujours accompagnée de graves manifestations fébriles, de caractère varié, de telle façon qu'avant de découvrir son étiologie, elle suscitait les diagnostics les plus divers : fièvre typhoïde, paludisme, cholécystite, néoplasie, colite, processus pleuro-pulmonaire, affection gastrique, affection hépatique, appendicite aiguë, entre autres hypothèses variées.

Cette difficulté de diagnostic doit être attribuée certainement au fait que la maladie ne figure pas encore dans les tableaux nosologiques du pays, étant donné l'imprécision et l'étendue de ses manifestations.

Pour la plupart des malades, le diagnostic avait comme fil conducteur une éosinophilie élevée, dans laquelle le maximum enregistré atteignait un pourcentage de 49,5 ; pour d'autres, ce furent les douleurs sourdes, localisées à l'hypochondre droit, la rate augmentée de

Ann, de Parasitologie, T. XXXI, N $1-2-1956$. 
volume, l'évolution lente et l'amaigrissement progressif qui ont permis de penser à la fasciolase.

Les globules blanes n'ont pas toujours été augmentés et n'ont pas dépassé un maximum de 17.800 par $\mathrm{mm}^{3}$ chez un des malades.

Chez tous, le diagnostic a été déterminé par la recherche positive d'œufs de Fasciola hepatica dans les selles et dans le suc duodénal obtenu par sondage avec du sulfate de magnésium. Les preuves de Takata-Ara, de Hanger et 'Timol n'ont pas toujours été positives et la courbe de l'électrophorèse (fig. 1), faite chez un des malades, a révélé un taux de globuline $\gamma$ augmenté. La bilirubinémie s'est révélée normale.

En ce qui concerne les conditions dans lesquelles les malades auraient été infectés, on doit certainement attribuer à la consommation de cresson cru, que tous mangeaient fréquemment, l'origine de la maladie.

Comme traitement, on a employé le chlorhydrate d'émétine, auquel tous les cas ont cédé, ce qui se vérifiait par une amélioration rapide avec absence de fièvre dès le quatrième jour de traitement.

L'atébrine essayée dans l'un de ces cas n'a révélé aucune action bienfaisante.

Pour un meilleur éclaircissement et complément de ce qui vient d'être exposé, nous présentons, dans le tableau I ci-après, le résumé des principales manifestations vérifiées et les résultats du traitement, aussi bien que l'évolution de l'éosinophilie dans le déroulement de la maladie, dans les cas que nous avons pu suivre de plus près.

Nous trouvons aussi intéressant de présenter l'histoire clinique de deux malades, curieuse soit par l'évolution de la maladie, soit par ses graves manifestations, soit par la diversité de diagnostics qu'ils ont suggérée.

$5^{\circ}$ CAs. - M.-M. B.-C., 27 ans, habitant à Santiago-do-Cacém (sud du Portugal). Tombe malade en janvier 1954, prise brusquement de frissons, de vertiges, de sueurs et de fièvre. Pendant une quinzaine de jours, la température oscille entre $38^{\circ} 5-39^{\circ}$, indifférente aux antibiotiques (pénicilline, streptomycine, terramycine), conduisant le praticien à admettre l'hypothèse d'une infection du groupe typhique.

L'état de la malade s'améliore, et elle s'en va pour Grandola, où elle présente un nouvel accès fébrile, traité par les chloromycétine et synergistine, avec quelque amélioration. Un examen radiologique du thorax n'a pas montré de lésion pleuro-pulmonaire. Au mois de février, elle vient à Lisbonne pour être mise en observation, et une nouvelle période fébrile apparait alors, atteignant la température de $39^{\circ}$, plus grave cette fois, à 
cause d'un abcès de la grande lèvre, qui oblige au drainage. Elle retourne chez elle en suivant le traitement, avec lequel elle n'a pas été améliorée. La malade souffre de troubles gastriques, de douleurs vagues de l'hypocondre droit, de vomissements et de température sub-fébrile. Les examens de sang révèlent une éosinophilie progressivement croissante. A la fin de mars, elle a des frissons, accompagnés de fièvre $\left(39^{\circ}-40^{\circ}\right)$, et on prescrit un traitement antipaludéen, sans résultat. Elle aime et consomme fréquemment du cresson cru.

Le 5 mai 1954 , elle vient à la consultation. L'examen objectif nous montre une malade angoissėe, amaigrie, sans indications cliniques anormales des appareils respiratoire et circulatoire. T. art. mx., 112 ; mn. 80. Abdomen : le rebord du foie se touche, dans les inspirations profondes, et se montre légèrement douloureux.

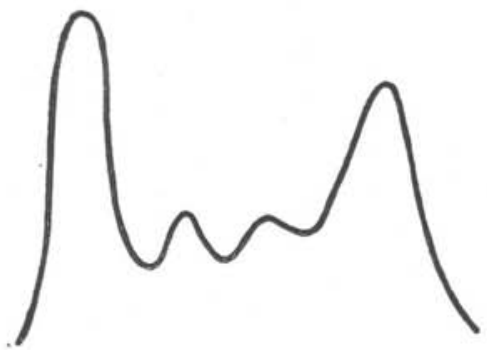

Fig. 1. - Courbe d'électrophorèse du malade $\mathrm{n}^{\circ} 6$.

Protéines totales : $7,8 \mathrm{gr}$. par $100 \mathrm{cc}$.

Albumine : $33 \%$ du total.

Alpha-globuline : $14 \%$ du total.

Bêta-globuline : $16,3 \%$ du total.

Gamma-globuline : $36,7 \%$ du total.

Dans la bile, recueillie par sondage duodénal, nous avons trouvé des œufs de Fasciola hepatica. On commence tout de suite un traitement à l'émétine, à raison de trois cures successives ( 0,2 gr. à chaque série), à dix jours d'intervalle, et, selon ce que nous dit notre collègue, le $\mathrm{D}^{\mathrm{r}}$ Alvès da Silva, de Santiago-do-Cacém, la malade est restée apyrétique au quatrième jour de traitement par l'émétine, et son état général s'est amélioré progressivement.

$6^{\circ}$ cas. - J. A.-M., 45 ans, marié, employé de commerce, né à Loulé (sud du Portugal).

Antécédents personnels : Paludisme à 5 ans. Syphilis à 31 ans, traitée au Néosalvarsan et bismuth pendant des années.

Antécédents héréditaires et collatéraux : Père décédé à 74 ans (ictus). Mère décédée à 67 ans (néoplasie maligne). Deux frères vivants et sains. Une sœur, morte de tuberculose pulmonaire. 


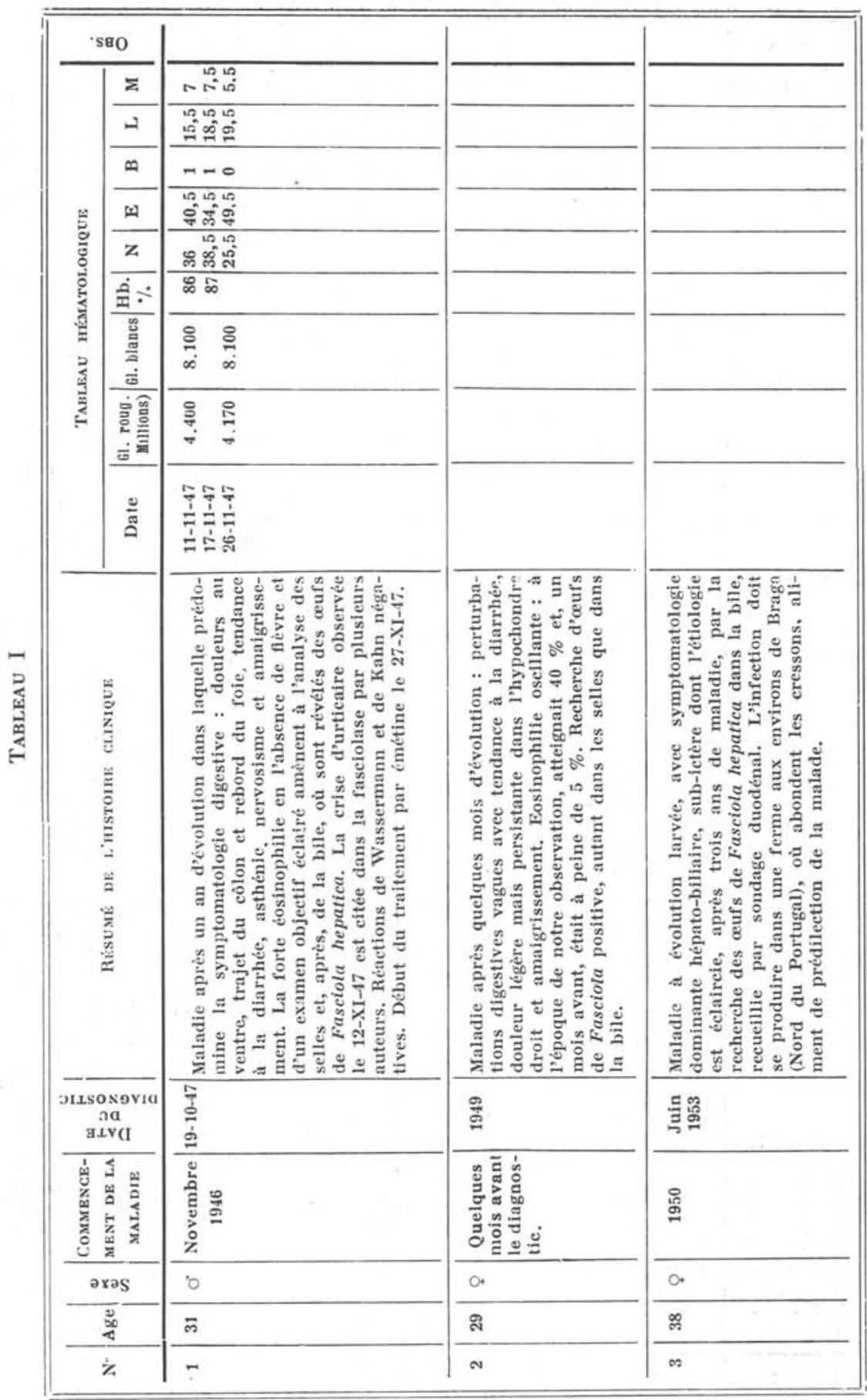

Ann. de Parasitologie, T. XXXI, No 1-2 - 1956. 


\begin{tabular}{|c|c|c|}
\hline 'sao & & \\
\hline$z$ & $\Rightarrow n$ & 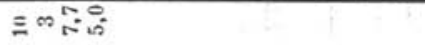 \\
\hline ـ & สิ- & 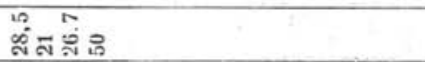 \\
\hline$\leadsto$ & oor & $0-=0$ \\
\hline$\omega$ & का $\infty$ & 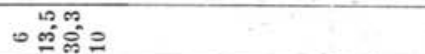 \\
\hline 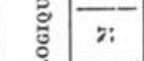 & ำ ำ & 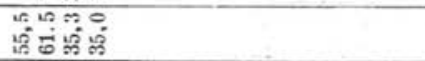 \\
\hline 安 & 웡 क & ミ8: \\
\hline 급 & 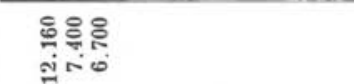 & 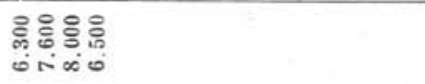 \\
\hline 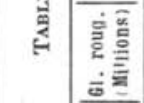 & 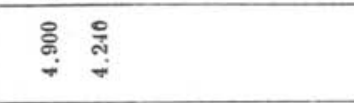 & 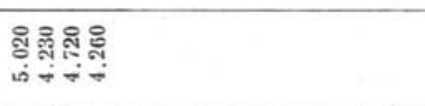 \\
\hline 气ّ & 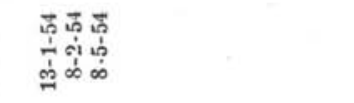 & 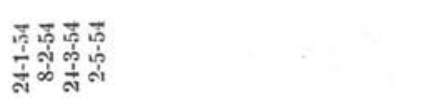 \\
\hline 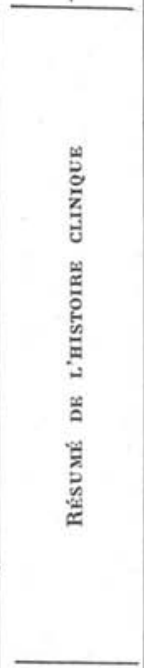 & 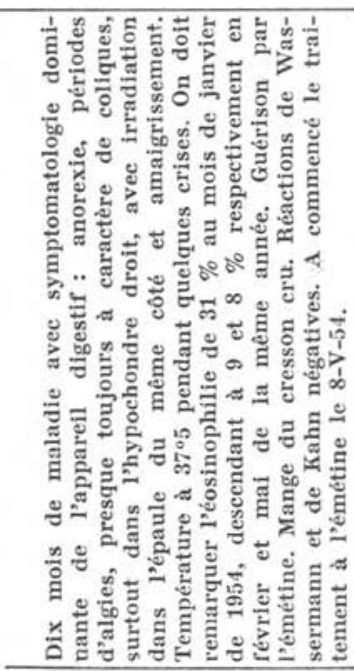 & 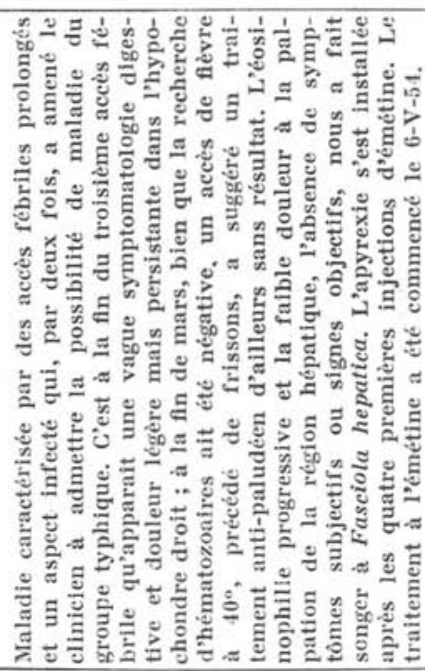 \\
\hline $\begin{array}{c}\text { Jusonovia } \\
\mathrm{ad} \\
\mathrm{gLV} \\
\end{array}$ & $\begin{array}{l}\text { 苟 } \\
\text { م⿱宀口 } \\
\infty\end{array}$ & 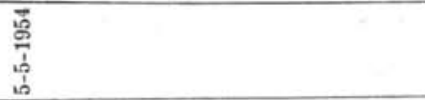 \\
\hline 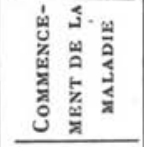 & 总骂 & 总苛 \\
\hline axas & o & 0. \\
\hline$\stackrel{0}{\infty}$ & क & ล \\
\hline$\dot{z}$ & + & 10 \\
\hline
\end{tabular}




\begin{tabular}{|c|c|c|c|}
\hline 'sao & & & \\
\hline \multirow{4}{*}{ 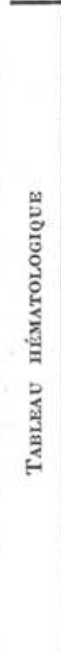 } & $\begin{array}{r}10 \% \\
+40 \\
-40 \\
-10\end{array}$ & กึ & $\rho^{\circ}$ \\
\hline & อ సิ & 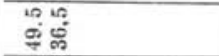 & $\stackrel{\infty}{\sim} \stackrel{0}{\sim}$ \\
\hline & $00 \stackrel{0}{0}$ & $\begin{array}{l}50 \\
=00\end{array}$ & on \\
\hline$\stackrel{1}{0}$ & 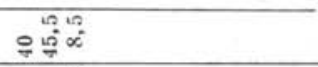 & $\stackrel{0}{i}-$ & ำ 구 \\
\hline 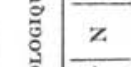 & ขำ & $\stackrel{5}{5}$ & $\approx$ 이 \\
\hline & ๓ 칳 흐 & $\infty 8$ & ప్రణ \\
\hline 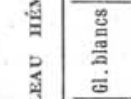 & 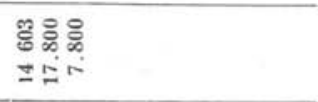 & 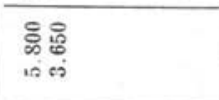 & $\begin{array}{l}\text { 웅유 } \\
\text { 용 }\end{array}$ \\
\hline 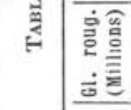 & 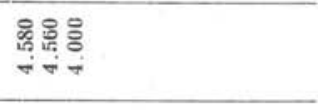 & 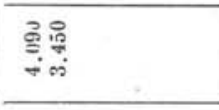 & $\begin{array}{l}\text { 윯ㅇ్తి } \\
\text {-is }\end{array}$ \\
\hline ڤัँ & 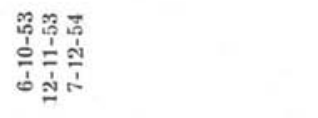 & 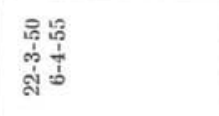 & 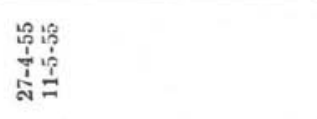 \\
\hline 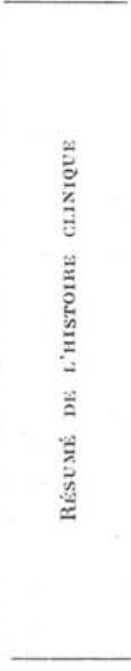 & 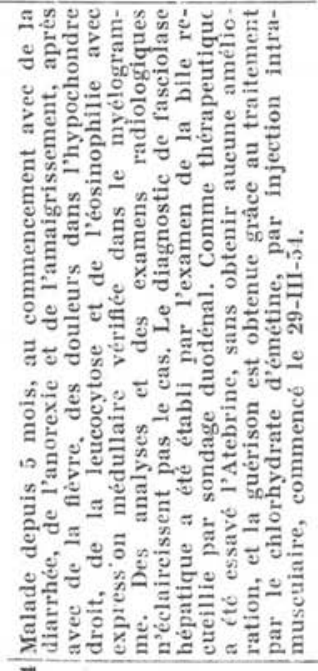 & 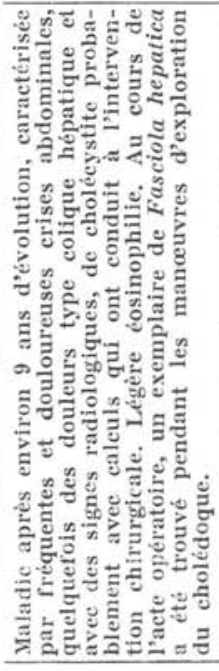 & 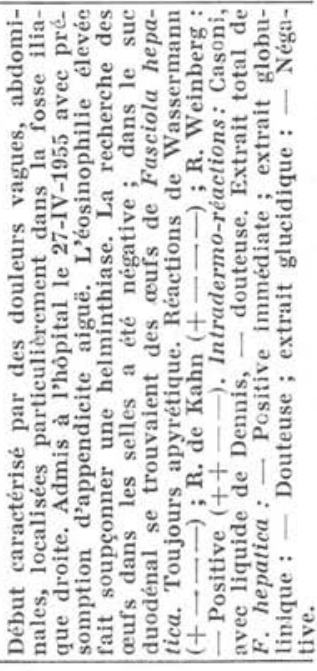 \\
\hline $\begin{array}{c}\text { JLSON9via } \\
\text { Ad } \\
\text { g.tvG }\end{array}$ & $\begin{array}{l}\text { 今̈ } \\
\text { ๙ै } \\
\text { తి }\end{array}$ & 呺 & $\begin{array}{l}: 0 \\
\vdots \\
\vdots \\
\dot{1}\end{array}$ \\
\hline 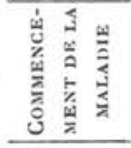 & 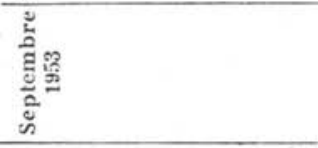 & 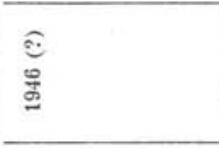 & 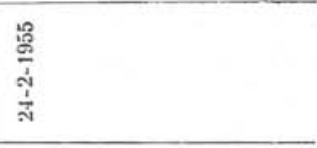 \\
\hline axas & 0 & o. & 0 \\
\hline 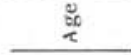 & 19 & कs & สี \\
\hline$\dot{z}$ & 0 & N & $\infty$ \\
\hline
\end{tabular}


H. A. : En septembre 1953, au retour de vacances, à Arcos-de-Valdevez (nord du Portugal), où il a mangé fréquemment du cresson cru, il a commencé avec une crise de diarrhée, ayant quotidiennement deux à trois selles liquides, sans mucosités, ni sang. Cet état s'est maintenu pendant environ vingt jours, accompagné d'anorexie et d'amaigrissement ; cependant, il a été soumis à l'observation du médecin, qui lui a prescrit le traitement au Ftalil K, qui l'a amélioré. Peu après, il se sent plus mal, avec des frissons et de la fièvre, ce qui lui fait prendre le lit, avec des douleurs dans le dos et dans l'hypocondre droit.

Le 6 octobre 1953 est fait un hémogramme, dont le résultat se trouve dans le tableau ci-joint. Ensuite, il est soumis à plusieurs examens de laboratoire et radiologique, en vue d'éclaircir la cause de l'éosinophilic.

Myélogramme (17 octobre 1953): Hémocytoblastes 1; myéloblastes 1,5 ; promyélocytes neutrophiles 2 ; myélocytes neutrophiles 2,5 ; métamyélocytes neutrophiles 3 ; granulocytes neutrophiles 10 ; promyélocytes éosinophiles 6,5 ; myélocytes éosinophiles 8 ; métamyélocytes éosinophiles 12 ; granulocytes éosinophiles 16 ; granulocytes basophiles 0,5 ; lymphocytes 2 ; monocytes 0,5 ; proérythroblastes basophiles 1 ; érythroblastes basophiles 9 ; érythroblastes polychromatophiles 13 ; érythroblastes orthochromatiques 6 ; mégacaryocytes 1 ; cellules non classifiées 4,5 $a a$, Pinto Nogueira.

Examen des urines (6 octobre 1953): Densité 1.020. Légères traces d'albumine. Cellules épithéliales polygonales et rondes. Rares leucocytes. Très rares hématies. Très rares cylindres hyalins.

Examen radiologique du thorax (7 octobre 1953) : Sans lésions pleuropulmonaires.

Cholécystographie: Cholécystopathie, due à l'augmentation du volume vésiculaire et déficit de concentration. Il n'y a pas de dyskinésie ni de calculs.

Rayons $\mathrm{X}$ d'estomac et duodénum : Diverticule de traction de la surface intérieure de l'œsophage au niveau du tiers médium. Sans quelques signes de lésion organique de l'estomac et duodénum. Hépatomégalie.

Pendant un mois, la température se maintient au voisinage de $38^{\circ} 5$ les premiers jours, avec des oscillations, et en descendant à $37^{\circ} 2$. Des antibiotiques ont été prescrits.

Le clinicien n'avait pas trouvé chez le malade d'autre symptomatologie que de l'amaigrissement et de la fièvre. A la fin de novembre, il a cessé toute thérapeutique, et depuis décembre le sujet poursuit sa vie avec difficulté, se sentant mal, asthénique, ayant perdu $13 \mathrm{~kg}$. dès le commencement de la maladie.

Nous avons observé le malade au mois de février 1954, et procédé à la recherche des œufs de Fasciola hepatica dans la bile obtenue par sondage duodénal; nous avons trouvé beaucoup d'œufs de ce parasite. Un traitement à l'atébrine a été institué, et le 29 mars 1954, comme il y avait encore des œufs de Fasciola dans la bile, on a commencé un traitement à l'émétine, à la dose de $0,04 \mathrm{gr}$. par jour, jusqu'à un total de 
0,4 gr. Un nouveau sondage duodénal, le 2 juin 1954, n'a pas révélé d'œufs de Fasciola hepatica dans la bile. Le malade s'améliore, recouvre l'appétit, augmente de poids, n'a plus l'aspect absorbé, angoissé, malheureux, qu'il présentait d'habitude à la consultation, il est plus confiant et plus optimiste. L'examen des appareils respiratoire, circulatoire et de l'abdomen n'a jamais montré d'altérations qui méritent d'être signalées.

\section{Discussion et conclusions}

L'occurrence récente de plusieurs cas humains de fasciolase hépatique au Portugal, au total 13, et dans d'autres pays, suscite un nouveau problème de santé publique, c'est-à-dire de défense humaine contre cette parasitose cosmopolite des herbivores.

En outre, il faut expliquer les raisons pour lesquelles cette parasitose, millénaire chez les herbivores, présente, il y a seulement quelques années, une incidence relativement élevée chez l'homme.

Comme problème sanitaire, il sera nécessaire surtout qu'on définisse et qu'on contrôle les aires de culture des cressons, principaux véhicules des métacercaires de la Fasciola hepatica, de façon à éviter leur infection par les œufs du parasite, à partir des herbivores, et qu'on combatte cette zoonose, soit par le traitement des animaux, soit par la lutte contre les mollusques, hôtes intermédiaires.

En ce qui concerne les motifs de l'incidence successivement croissante de la fasciolase humaine dans le globe, il ne nous semble pas très facile de donner du fait une explication complète. Elle pourra être attribuée à un accroissement de la fasciolase chez les herbivores ou à l'augmentation de la consommation du cresson cru, ou elle pourra être la conséquence de l'adaptation biologique plus parfaite du parasite chez l'homme, d'où résulte la possibilité de son évolution chez lui jusqu'à la phase adulte.

Nous devons signaler que l'exemplaire que nous avons obtenu dans notre cas $\mathrm{n}^{\circ} 7$ présentait les caractéristiques morphologiques des exemplaires récoltés chez les herbivores. De même, les œufs que nous avons observés, soit dans les selles, soit dans le suc duodénal, sont indistinguables de ceux qui sont éliminés par les hôtes naturels du parasite, ce qui nous permet de conclure que, tout au moins apparemment, l'organisme humain est parfaitement favorable au parasite.

Nous attendons que les études se poursuivent sur le sujet, de façon à déterminer la position du parasite en présence de l'homme et aussi pour que soient mieux établies les bases de la défense de l'homme contre cette distomatose dont la fréquence est en constante augmentation dans le monde. 


\section{BIBLIOGRAPHIE}

Aguiló (F. de S.), 1953. - Una nueva helmintiasis humana en Colombia : la fascioliasis hepatica. Rev. Ac. Col. Ciencias Exactas, Fisicas y Naturales, 9 (33/34), 2133/34. Helm. Abstracts, 22, 135, 80,

Brumpt (E.), 1910. - Précis de Parasitologie, Paris.

Deschiens (R.), 1952. - Distomatoses hépatiques. In VAucel (M.), Médecine Tropicale, Paris.

Ehlers (H. J.) \& Knuttgen (H.), 1949, 1950. - Ein Fall von Distomatosis hepatica bei einem $81 / 2$ jährigen Mädchen. Ztsch. f. Tropenmed. u. Parusit., 1, 3, 364. In Trop. Dis. Bull., 47, 7, 638.

Fonseca (F.) e Fraga de Azevedo (J.), 1948. - Un cas humain de fasciolase hépatique. Ann. Par. Hum. et Comp., 23, 18.

- da Gama (M.) e Branco (F.), 1952. - Eosinofilia elevada. Comunicação ão Congresso Nacional de Med. Trop.

Gradwohl (R. B. H.) and Kouri (P.), 1948. - Clinical laboratory Methods and Diagnosis. vol. III. Parasitology and Tropical Medicine, Saint-Louis.

Hoffman (W. A.) \& Rivera (T.), 1929, 1930. - The precipitin test in Fascio?a hepatica Infection, Porto-Rico. Rev. of Public Health \& Trop. Med., 4, 12, 589. In Trop Dis. Bull., 27, 6, 452.

Laviér (G.) et Stefanopoulo (G.), 1944. - L'intradermo-réaction et la réaction de fixation du complément dans la distomatose humaine à Fasciola hepatica. Bull. Soc. Path. Exot., 37, 302.

LAvier (G.), 1945. — La valeur diagnostique du taux de l'éosinophilie sanguine dans les helminthiases. Presse Médicale, 49, 535.

MAIA (C.), 1952. - Terapéutica radical de duas parasitoses raras (Fasciolase hepática e balantidiase intestinal). Ann. Inst. Med. Trop., 9, 4, 1475.

Mara (C.), 1951. - A propósito de cinco casos humanos de Fasciolase hepática. Jornal do Médico, 18, 49.

Marin (J.), Diaz y Ramirez (Al.), 1951. - Un nuevo caso de hallazgo cropologico de huevos de Fasciola. La. Med. Col., 17, 164.

MinNing (W.) \& VoGEL (H.), 1950. - Immunbiologische und epidemiologische untersuchungen bei 3 Fällen von menschlicher Fasciolose. Ztschr. $f$. Tropenmed. u. Parasit., 1, 4, 532. In Trop. Dis. Bull., 47, 9, 863.

Монr (W.), Berka (W.), Knuttgen (H.) \& OHr (A.), 1951, 1952. - Das Klinische Bild der Distomatosis hepatica (Fasciola hepatica) und ihre Terapie. Med. Monatsschr., octobre, $\mathrm{n}^{\circ}$ 10, 676. In Trop. Dist. Bull., 49, 2, 159.

Moormann (H.), 1950. - Die Leberegelkrankheit (Fasciolosis) beim Menschen. Eine Klinische Betrachtung mit Kasuistischen Beitrag (Beobachtung einer Fasciolosis bei einem Kinde). Med. Klin., 45, 1, 4-8. In Trop. Dis. Bull., 47, 5, 478.

Murphy (F. D.) \& Pascall (K. G.), 1950. - Liver Fluke in the Common Bile Duct. Brit. Med. J., Mar., 18, 647.

Senevet (G.) et Champagne (R.), 1929. - A propos d'un cas de distomatose à Fasciola hepatica. Arch. Inst. Past. d'Algérie, 7, 207.

VILAS (J. M. T.), 1954. - Las zoonosis parasitarias transmisibles al hombre en el Somontano de Barbastro, Barbastro. 